\title{
Análisis de metodologías para desarrollar Data Warehouse aplicado a la toma de decisiones
}

\section{Analysis of methodologies to develop Data Warehouse applied to decision making}

Geovanny Euclides Silva Peñafiel. ${ }^{1}$, Verónica Marcela Zapata Yánez. ${ }^{2}$, Morales Guamán Klever Patricio. ${ }^{3}$ \& Toaquiza Padilla Luis Marcelo. ${ }^{4}$

Recibido: 15-07-2019 / Revisado: 25-07-2019 /Aceptado: 20-08-2019/ Publicado: 10-09-2019

\begin{abstract}
.
DOI: https://doi.org/10.33262/cienciadigital.v3i3.4..922

The study follows the analysis of methodologies to develop a data warehouse for decision making, which facilitates the selection of a suitable methodology for the management of the data. the externalization of the data will be presented in a qualitative way, which will serve as support for the identification of the important factors that should be assessed. Due to the nature of the explanatory descriptive study, it will rely on the development of methodological prototypes with the proposals made by Ralph Kimball, Hefestos 2.0 and SAS Rapid Data Warehouse Methodology; through the analysis of both the characteristics and the phases that integrate the methodologies; For this, parameters such as: the degree of compliance with the objectives, the capacity for improvement and the degree of satisfaction of managers and employees in the analysis, design and construction of a Data Warehouse for decision-making were taken. The study has obtained results that determine a robust foundation for the Hefestos version 2.0 methodology; due to the high percentage acquired in the 6 phases examined, with its 54 points, while, in the analysis of characteristics, 29 points are obtained for Hefestos. The use of the Hefestos methodology, allowed to quickly identify and understand the objectives that the institution wanted to achieve with the implementation of the Data Warehouse, helped to determine the needs when establishing the requirements based on the user; involving it in each stage of the information process and determining the ideal data for decision making.
\end{abstract}

Keywords: Data warehouse, methodologies, Hefestos 2.0, Kimball, SAS Rapid.

\footnotetext{
${ }^{1}$ Pontificia Universidad Católica del Ecuador Sede Ambato,Ecuador, geova3006sp@yahoo.es

${ }^{2}$ Pontificia Universidad Católica del Ecuador Sede Ambato Ecuador, verozy26@gmail.com

${ }^{3}$ Pontificia Universidad Católica del Ecuador Sede Ambato Ecuador, kmorales.tech@gmail.com

${ }^{4}$ Pontificia Universidad Católica del Ecuador Sede Ambato Ecuador, amarcetp@gmail.com
} 


\section{Resumen.}

La investigación persigue el análisis de metodologías para desarrollar un Data Warehouse para la toma de decisiones; que permita la selección de la metodología adecuada para el manejo de los datos, la exteriorización de los datos se presentará de forma cualitativa, los cuales servirán de soporte para la identificación de los factores importantes que deben ser valorados. Debido a la naturaleza del estudio de tipo descriptiva explicativa, se apoyará en el desarrollo de prototipos metodológicos con las propuestas realizadas por Ralph Kimball, Hefestos 2.0 y SAS Rapid Data Warehouse Methodology; por medio del análisis tanto de las características como de las fases que integran las metodologías; para ello se tomaron parámetros tales como: el grado del cumplimiento de los objetivos, la capacidad de mejoras y del grado de satisfacción de los directivos y empleados en el análisis, diseño y construcción de un Data Warehouse para la toma de decisiones. Del estudio se ha obtenido resultados que determinan una cimentación robusta para la metodología Hefestos versión 2.0; debido al alto porcentaje adquirido en las 6 fases examinadas, con sus 54 puntos, mientras que, en el análisis de características se obtienen 29 puntos para Hefestos. El uso de la metodología Hefestos, permitió identificar ágilmente y comprender los objetivos que la institución deseaba alcanzar con la implementación del Data Warehouse, ayudó a determinar las necesidades al establecer en los requerimientos en base al usuario; involucrándolo en cada etapa del proceso de información y determinar los datos idóneos para la toma de decisiones.

Palabras claves: Data Warehouse, metodologías, Hefestos 2.0, Kimball, SAS Rapid.

\section{Introducción.}

Tradicionalmente se considera que el éxito o fracaso de cualquier política o plan estratégico aplicado a una empresa depende de que sus actores tengan una total comprensión del sistema al cual tratan de influenciar; pero las organizaciones y empresas financieras y comerciales han enfocado todos su esfuerzos a proveer sistemas de información transaccionales y a la generación de datos sobre todos los aspectos de sus actividades diarias, pero sólo una pequeña fracción de los datos capturados, procesados y almacenados en la empresa está realmente disponible para ejecutivos y tomadores de decisiones; es hasta la actualidad que las compañías han podido explotar esta información en la toma de decisiones, otorgando mayor rentabilidad a todas las acciones realizadas dentro de la empresa.

Debido al tamaño y la complejidad de los datos que los responsables de las decisiones deben manejar en diferentes empresas, es muy común que los efectos de esta toma de decisiones resulte inadecuados o contraproducentes cuando se trata de lograr productividad para la empresa; es por ello que el desarrollo de los métodos de análisis de datos, han permitido la optimización en la obtención de la información, toma de decisiones oportunas en el momento 
adecuado, lo que genera una minimización de costos, tiempo y recursos, este fenómeno se relaciona de forma directa con la inteligencia de negocio.

Las soluciones Data Warehousing han sido diseñadas para que evolucionen y se desarrollen en base a los requerimientos del negocio, donde la información varía de acuerdo a un período de tiempo; por el contrario de lo que nos ofrecen los sistemas operacionales.

Existen diferentes metodologías que permiten la implementación de almacenes de datos, para su posterior análisis actual e histórico, que permita a sus actores conocer la situación de una empresa en base a indicadores, con la finalidad de tomar una decisión acertada.

Por lo que se ha visto la necesidad con el presente estudio orientar al análisis de metodologías con el propósito de determinar la óptima para la construcción de un repositorio que permita seguir con el procedimiento de control y tratamiento respectivo de la información.

Al analizar las fases y los procesos que se realizan en cada una de las metodologías en el desarrollo del Data Warehouse, se acopló a Hefesto versión 2.0 que es una metodología propia para la construcción de Data Warehouse, que garantiza mayores prestaciones, certificando los datos para la toma de decisiones.

\section{Desarrollo}

\section{Inteligencia de negocios}

La inteligencia de negocios apoya a la toma de decisiones del negocio: estratégicas, tácticas y operativas; según varios autores:

"Business Intelligence es el proceso de convertir datos en conocimiento, y el conocimiento en acción, para la toma de decisiones" (Bernabeu R. , 2010).

Desde un punto de vista pragmático, y asociándolo directamente con las tecnologías de la información, se define a Business Intelligence como "el conjunto de metodologías, aplicaciones y tecnologías que permiten reunir, depurar y transformar datos de los sistemas transaccionales e información desestructurada (interna y externa de la compañía) en información estructurada, para su explotación directa (reporting, análisis OLTP/OLAP, alertas) o para su análisis y conversión en conocimiento dando así soporte a la toma de decisiones sobre el negocio" (Sinnexus, 2016).

Entre sus características destacan:

Accesibilidad a la información: se debe garantizar el acceso de forma independiente de los usuarios sin tener en cuenta la procedencia de los datos. 
- Apoyo a la toma de decisiones: permite la manipulación de la información por parte de los usuarios de manera que estos los conjuguen con las herramientas de análisis y les permitan realizar una toma de decisiones acertada

- Orientación al usuario final: se busca la independencia entre conocimientos técnicos de usuarios y su capacidad para utilizar estas herramientas.

\section{Datos, información y conocimiento}

La forma apropiada para diferenciar los términos; los datos están localizados en el mundo y el conocimiento se encuentra ubicado en agentes de cualquier tipo, mientras que la información adopta un papel mediador entre ambos.

Figura 1. Datos, información y conocimiento

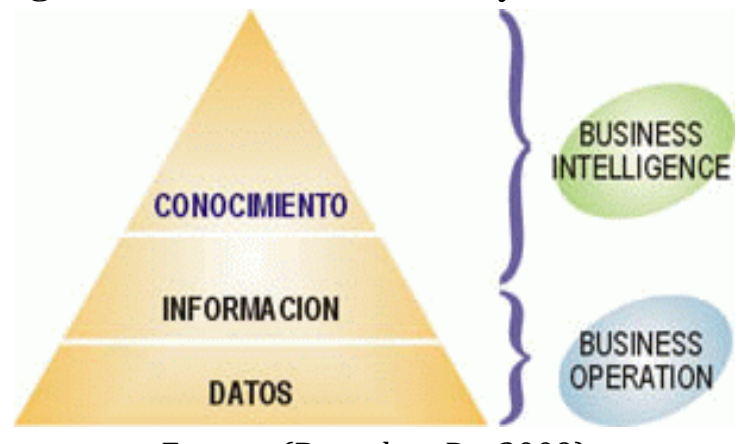

Fuente: (Bernabeu D. , 2009)

\section{Datos}

Se consideran elementos primarios de la información cuyo valor por si solos no son de relevancia para la toma de decisiones, son la mínima unidad semántica; los datos pueden ser una colección de hechos almacenados en algún dispositivo; pueden ser internos o externos a la organización, objetivos o subjetivos o de tipo cualitativos y cuantitativos (Turnero, 2015).

\section{Información}

Un conjunto de datos procesados con un significado, un propósito y/o contexto; cuya utilidad reside en la toma de decisiones debido a la minimización de la incertidumbre, para la transformación en información de los datos se les añade valor (Computación, 2010):

- Contextualizar: contexto y propósito de su generación

- Categorizar: se conocen las unidades de medida que ayudan a interpretarlos.

- Calcular: procesamiento matemático o estadístico

- Corregir: depuración de los datos; errores e inconsistencias

- Condensar: resumen de datos

Información $=$ Datos + Contexto (añadir valor $)+$ Utilidad (disminuir la incertidumbre $)$ 


\section{Conocimiento}

El conocimiento es la combinación de experiencias, valores, información y "saber hacer" que sirve como marco para la incorporación de nuevas experiencias e información, y es útil para la acción; para que la información se transforme en conocimiento es necesario realizar (Vicente, 2017):

- Comparación con otros elementos

- Predicción de consecuencias

- Búsqueda de conexiones

- Conversación con otros portadores de conocimiento.

\section{Arquitectura típica de soluciones Business Intelligence}

Las tres capas básicas de este tipo de soluciones son: BDD Relacional, BDD Multidimensional y herramientas de visualización.

Figura 2. Arquitectura de una solución BI

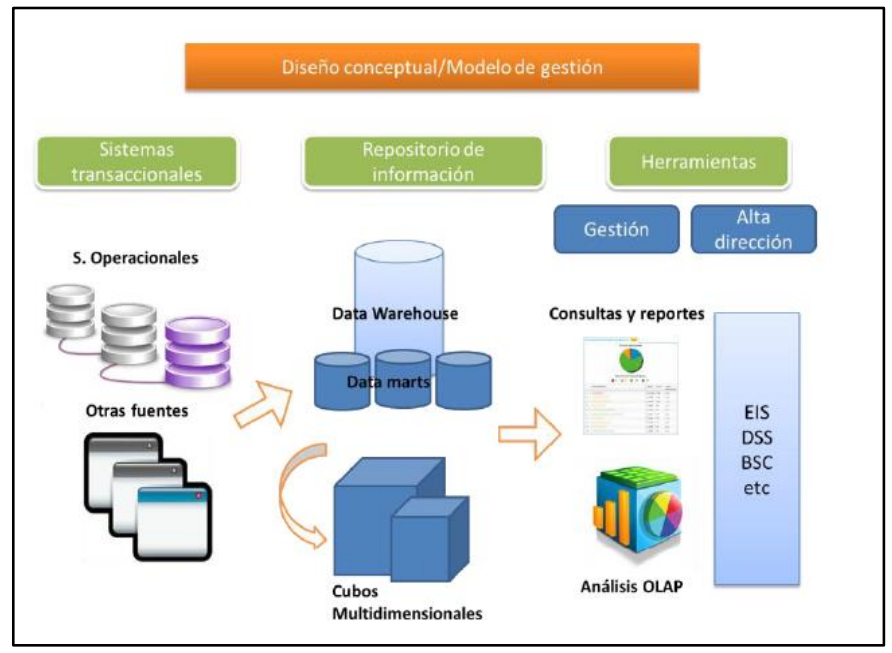

Fuente: (Villareal, 2013)

\section{Funciones}

Tabla 1: Funciones de los componentes del Business Intelligence

\begin{tabular}{|c|c|c|}
\hline Base de datos relacional & $\begin{array}{c}\text { Base de datos } \\
\text { multidimensional }\end{array}$ & Visualizado \\
\hline $\begin{array}{l}\text { Depuración } \\
\text { homogeneización } \\
\text { Almacenamiento de datos } \\
\text { Motos de cálculo, solo de ser } \\
\text { necesario }\end{array}$ & $\begin{array}{l}\text { Velocidad de acceso y consulta } \\
\text { Capacidad de análisis desde } \\
\text { varios puntos de vista } \\
\text { (dimensiones de análisis) }\end{array}$ & $\begin{array}{l}\text { Análisis de la información } \\
\text { (Drill-down, drill-through y } \\
\text { rotación de filas y columnas) }\end{array}$ \\
\hline
\end{tabular}

Fuente: Elaboración propia 


\section{Data Warehousing}

"Un Data Warehounsing es un almacén único, completo y consistente de datos obtenidos de una variedad de fuentes y puestos a disposición de los usuarios finales de una manera que puedan entender y utilizar en un contexto empresarial" (Cuellar, 2011).

"El Data Warehounsing es un proceso, no un producto, para ensamblar y administrar datos de diversas fuentes con el fin de obtener una visión única y detallada de una parte o de toda una empresa" (Devlin, 2011).

De lo mencionado por los autores citados anteriormente se puede concluir que el Data Warehousing es el proceso mediante el cual los datos son transformados y agrupados dentro de un almacén de datos., permitiendo una fusión de los mismos los cuales provienen de diversas fuentes hacia un solo contexto, lo cual permite un acceso rápido a la información y por consiguiente a la toma de decisiones

\section{Data Warehouse}

En el contexto informático, Data Warehouse en español almacén de datos es una colección de información corporativa, derivada directamente del sistema operativo y algunas fuentes externas de datos, su propósito específico es apoyar las decisiones empresariales. Se trata de un expediente completo de una empresa, más allá de la información transaccional y operacional, almacenada en una base de datos, diseñada para favorecer el análisis y la divulgación eficiente de datos (Peña J \& Súarez, 2008).

Figura 3. Arquitectura de un Data Warehouse

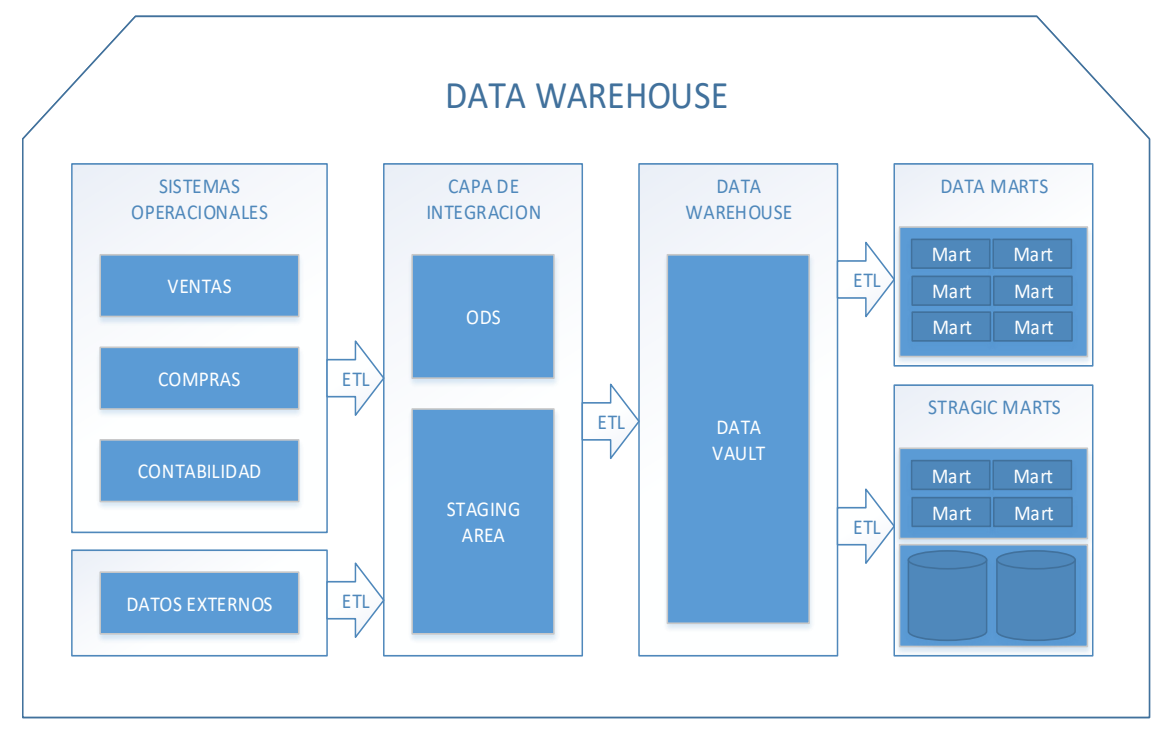

Fuente: (Villareal, 2013) 


\section{Bill Inmon}

Bill Inmon fue uno de los primeros autores en escribir sobre el tema de los almacenes de datos, para él es necesaria la trasferencia de la información de los OLTP de la empresa a un solo lugar, centralizando los datos para su análisis (CIF o Corporate Information Factory) el cual debe cumplir con las características de un repositorio de datos:

- Orientado a temas. - Los datos y elementos concernientes a un mismo tema, deben estar organizados de tal manera que ellos queden unidos entre si dentro del almacén de datos

- Variante en el tiempo. - Los cambios producidos en los datos a lo largo del tiempo quedan registrados para que los informes que se puedan generar, reflejen esas variaciones.

- No volátil. - La información no se modifica ni se elimina, una vez almacenado un dato, éste se convierte en información de sólo lectura, y se mantiene para futuras consultas.

- Integrado. - La base de datos contiene los datos de todos los sistemas operacionales de la organización, y dichos datos deben ser consistentes.

Figura 4. Arquitectura de un Data Warehouse según Bill Inmon

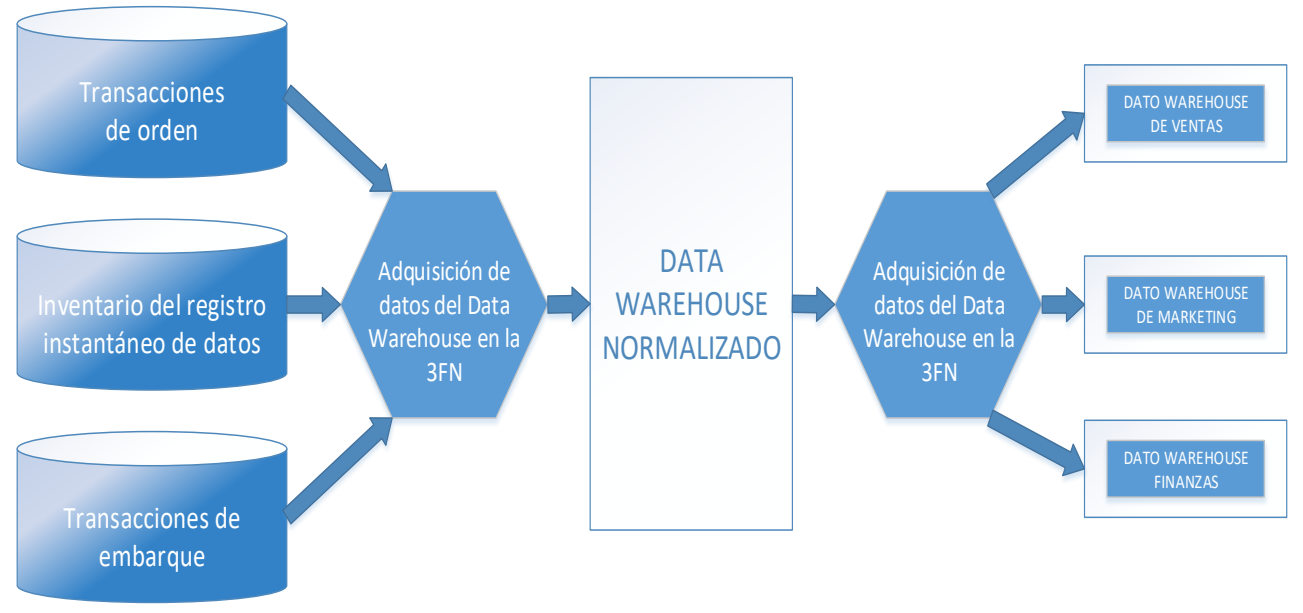

Fuente: (Espinosa, 2010)

Inmon defiende una metodología descendente (top-down) a la hora de diseñar un almacén de datos, ya que de esta forma se considerarán mejor todos los datos corporativos. En esta metodología los Data Marts se crearán después de haber terminado el Data Warehouse completo de la organización (Kimball, 2016).

El análisis de las metodologías para el desarrollo de un Data Warehouse conlleva estudio del conjunto de procedimientos que se siguen para construir un sistema, un ciclo de vida de 
desarrollo de sistema (SDLC, Systems Development Life Cycle). Entre las metodologías se analizarán las siguientes.

\section{Metodología Ralph Kimball}

\section{Introducción}

La metodología de Kimball, llamada Modelo Dimensional (Dimensional Modeling), se basa en lo que se denomina Ciclo de Vida Dimensional del Negocio (Business Dimensional Lifecycle). Esta metodología es considerada una de las técnicas favoritas a la hora de construir un Data Warehouse.

Un almacén de datos (Data Warehouse) es una colección o conglomerado de datos o Data Marts orientada a un determinado ámbito (empresa, organización, etc.), integrado, no volátil y variable en el tiempo, que ayuda a la toma de decisiones en la entidad en la que se utiliza. Es una estructura de datos donde la información contenida está diseñada para favorecer el análisis y la divulgación eficiente de datos.

En el Modelo Dimensional se constituyen modelos de tablas y relaciones con el propósito de optimizar la toma de decisiones, con base en las consultas hechas en una base de datos relacional que están ligadas con la medición o un conjunto de mediciones de los resultados de los procesos de negocio.

El Modelo Dimensional es una técnica de diseño lógico que tiene como objetivo presentar los datos dentro de un marco de trabajo estándar e intuitivo, para permitir su acceso con un alto rendimiento (León, Ciclo de vida de Ralph Kimball, 2014).

\section{Características}

Este ciclo de vida del proyecto de DW, está basado en cuatro principios básicos:

- Centrarse en el negocio: Hay que concentrarse en la identificación de los requerimientos del negocio y su valor asociado, y usar estos esfuerzos para desarrollar relaciones sólidas con el negocio, agudizando el análisis del mismo y la competencia consultiva de los implementadores.

- Construir una infraestructura de información adecuada: Diseñar una base de información única, integrada, fácil de usar, de alto rendimiento donde se reflejará la amplia gama de requerimientos de negocio identificados en la empresa.

- Realizar entregas en incrementos significativos: crear el almacén de datos (DW) en incrementos entregables en plazos de 6 a 12 meses. Hay que usa el valor de negocio de 
cada elemento identificado para determinar el orden de aplicación de los incrementos. En esto la metodología se parece a las metodologías ágiles de construcción de software.

- Ofrecer la solución completa: proporcionar todos los elementos necesarios para entregar valor a los usuarios de negocios. Para comenzar, esto significa tener un almacén de datos sólido, bien diseñado, con calidad probada, y accesible. También se deberá entregar herramientas de consulta ad hoc, aplicaciones para informes y análisis avanzado, capacitación, soporte, sitio web y documentación.

\section{Fases}

Las fases establecidas por Ralph Kimball, han sido diseñadas para que puedan ser desarrolladas en paralelo o en forma secuencial; cada una de las fases planteadas en esta metodología garantiza la calidad en el desarrollo del Data Warehouse

Figura 5. Fases Metodología Ralph Kimball

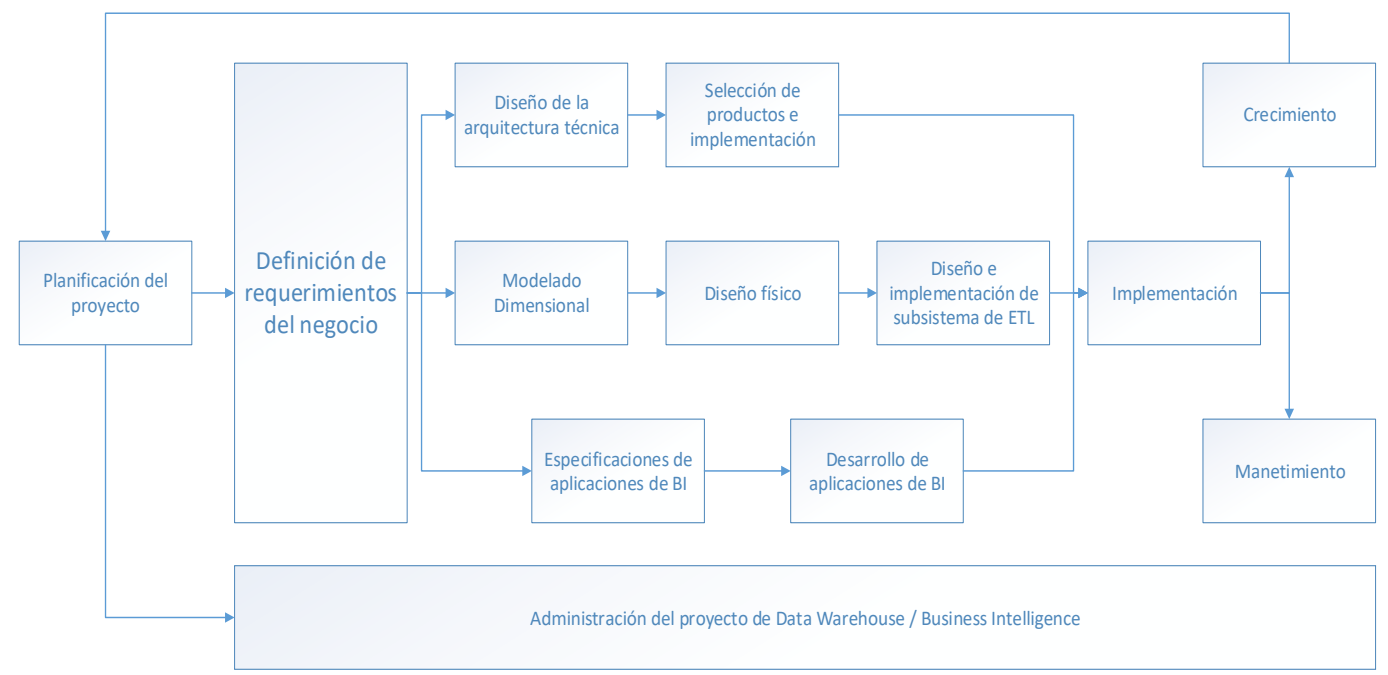

Fuente: (Murillo, 2008)

\section{Metodología Hefestos}

\section{Introducción}

Esta metodología se fundamenta en una amplia investigación y comparación entre metodologías existentes, además de expectativas propias del autor en procesos de desarrollo de almacenes de datos, la cual está en continua evolución y que toma en cuanta, como valor agregado, todas las aportaciones de la gran comunidad que la utiliza (Bernabeu R., 2010).

Para la implementación de un Data Warehouse se debe tener en cuenta que las fases de la metodología no sean muy extensas, ni compliquen el desarrollo del mismo; es por ello que 
para Hefestos se requiere de cuatro pasos: Análisis de requerimientos, Análisis de los OLTP, Modelo lógico del Data Warehouse e Integración de datos; con esto logramos establecer las necesidades primordiales de la información por parte de los actores del sistema, e identificar las fuentes de datos de forma concreta y sus indicadores, y la creación de modelo de datos.

\section{Características}

La metodología de Hefestos posee las características listadas a continuación:

- En cada una de las fases se puede distinguir fácilmente los objetivos que se persiguen, así como los resultados esperados; adicionalmente son de fácil comprensión

- La estructura del Data Warehouse es de fácil y rápida adaptación, esto se debe a que fue construida en base a los requerimientos de los usuarios.

- La resistencia que presentan los usuarios finales al cambio se ve reducido gracias a que en cada etapa se los considera para determinar el comportamiento y las funciones que se incorporan al diseño del DW

- Los modelos conceptuales y lógicos que se implementan son de sencilla comprensión y análisis.

- El tipo de ciclo de vida que contenga a la metodología marcan independencia el uno del otro.

- Las herramientas que se utilicen para la construcción del DW son independientes de la metodología

- La metodología es independiente de las estructuras físicas y su distribución, que contengan el DW.

- Los resultados obtenidos al finalizar una fase se convierten en un nuevo punto inicial para el siguiente paso.

- Se aplica tanto para el Data Warehouse como para los Data Mart

\section{Fases}

Figura 6. Fases Metodología Hefesto

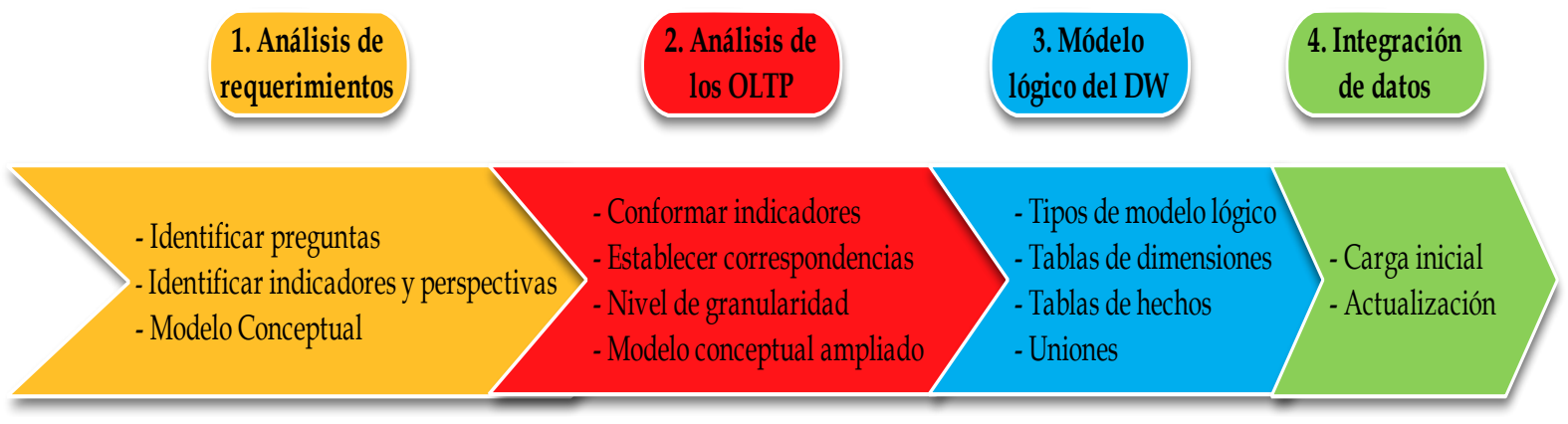

Fuente: elaboración propia 


\section{SAS Rapid Data Warehouse Methodology}

\section{Introducción}

Siendo la construcción de un Data Warehouse un proceso evolutivo, este tiene que apoyarse en una metodología, la cual establezca sus fases en el alcance del proyecto; el seguir los pasos de la metodología y comenzar el desarrollo del Data Warehouse desde un área específica de una empresa, nos permite obtener resultados tangibles y minimiza el tiempo de entrega.

\section{Fases}

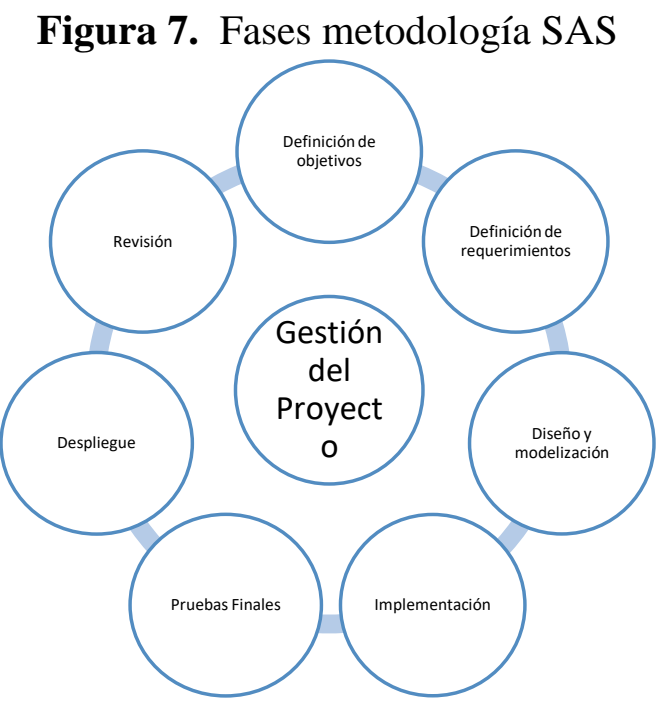

Fuente: (Fernández, 2015)

\section{Metodologia.}

El estudio realizado es de tipo descriptiva explicativa, ya que involucra la evaluación de las fases y actividades de cada una de las metodologías seleccionadas.

La exteriorización de los datos se presentará de forma cualitativa lo cual ayudarán a la identificación de los factores importantes que deben ser valorados, para lo cual se manejará la escala de Likert en la ejecución del estudio comparativo (REDIFP, 2013).

En esta investigación se tendrá como ambiente operativo del Data Warehouse, esto es: almacenamiento, consolidación y extracción de información de diversas fuentes, para tratarla, permitiendo su exploración e investigación, desde diferentes perspectivas, facilitando la toma de decisiones, además se utilizará el método descriptivo y el método comparativo.

Parámetros de comparación de las fases metodológicas: 
Tabla 2: Parámetros de comparación de fases metodológicas

\begin{tabular}{|c|c|}
\hline Indicadores & Índices \\
\hline Fase 1: Requerimentación & $\begin{array}{l}\text { Determinación de requerimientos } \\
\text { Características de la organización } \\
\text { Análisis de usuarios }\end{array}$ \\
\hline Fase 2: Estrategia de proyecto & $\begin{array}{l}\text { Cuantificar el tiempo para cumplir la necesidad (corto- } \\
\text { mediano-largo plazo) } \\
\text { Definir ventajas y desventajas }\end{array}$ \\
\hline Fase 3: Planificación del proyecto & $\begin{array}{l}\text { Cronograma de actividades } \\
\text { Análisis de riesgo } \\
\text { Definición de responsabilidades y grupo de trabajo }\end{array}$ \\
\hline Fase 4: Selección de la tecnología & $\begin{array}{l}\text { Entorno actual tecnológico de la organización } \\
\text { Definición de tecnologías }\end{array}$ \\
\hline $\begin{array}{l}\text { Fase 5: Diseño del sistema de } \\
\text { información } \\
\text { Fase 6: Elaboración del sistema } \\
\text { de información }\end{array}$ & $\begin{array}{l}\text { Determinación del modelo de información } \\
\text { Diseño de la interfaz de usuario } \\
\text { Análisis de requerimientos (indicadores) } \\
\text { Modelo conceptual } \\
\text { Análisis OLTP } \\
\text { Conformar indicadores } \\
\text { Nivel de granularidad } \\
\text { Nivel conceptual ampliado } \\
\text { Modelo lógico de la estructura del DW } \\
\text { Tablas de dimensiones } \\
\text { Tablas de hechos } \\
\text { Procesos ETL }\end{array}$ \\
\hline
\end{tabular}

Fuente: (Garcés, 2015)

La población es el conjunto de elementos a ser evaluados, la población constituye las metodologías de desarrollo del Data Warehouse y los estándares para el desarrollo de este.

La muestra se encuentra determinada por una selección no probabilística de las metodologías de desarrollo para Data Warehouse desarrollado en el Estudio Comparativo de Metodologías e implementación de alternativas BI open source vs propietarias en entornos tradicionales de la Ingeniera Magdalena Garcés en el cual se ha determinado que las metodologías Hefestos, Kimbal y SAS Methodology son las óptimas para la implementación de un proyecto BI.

\section{Resultados}

Determinación de parámetros de comparación fase metodológica

Para la identificación de la metodología que respalde el alcance de las metas planteadas para es necesario realizar un estudio descriptivo-explicativo de sus fases y actividades involucradas, para ello se elabora una lista de todas las tareas que las metodologías elegidas efectúan, y se agruparon sus criterios en 6 fases. 
Usando la escala de Likert para la respectiva demostración, tomando los valores previamente estudiados, siendo:

Tabla 3: Escala de Likert

\begin{tabular}{cc}
\hline Escala & Valoración \\
\hline Baja & 1 \\
Media & 2 \\
Alta & 3 \\
\hline \multicolumn{2}{c}{ Fuente: elaboración propia }
\end{tabular}

\section{Fase 1. Requerimentación}

Tabla 2: Resumen. Indicador requerimientos

\begin{tabular}{lccc}
\hline \multirow{2}{*}{\multicolumn{1}{c}{ Indicador }} & \multicolumn{3}{c}{$\begin{array}{c}\text { Metodologías desarrollo de Data } \\
\text { Warehouse }\end{array}$} \\
\cline { 2 - 4 } & Kimball & Hefesto & SAS \\
\hline Determinación de requerimientos & 2 & 3 & 2 \\
Características de la organización & 2 & 3 & 2 \\
Análisis de usuarios & 3 & 1 & 1 \\
TOTAL & $\mathbf{7}$ & $\mathbf{7}$ & $\mathbf{5}$ \\
\hline
\end{tabular}

Fuente: elaboración propia

Gráfico 8. Resumen. Indicador Requerimientos

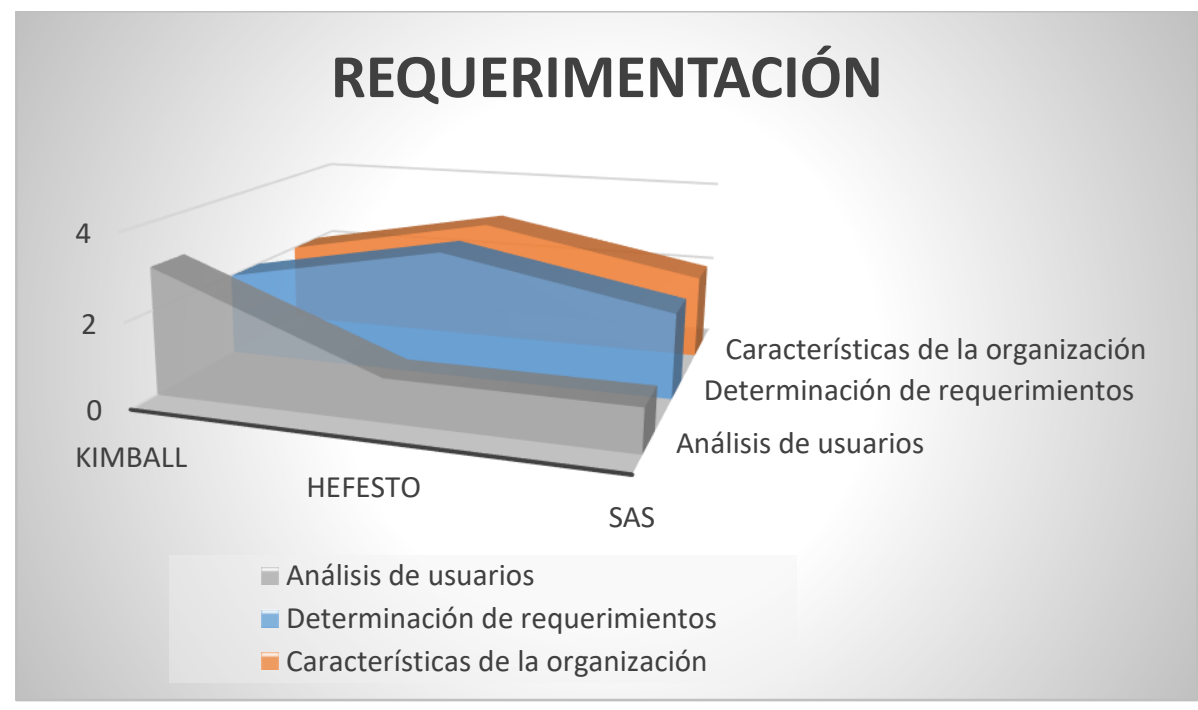

Fuente: Elaboración propia 


\section{Fase 2: Estrategia de proyecto}

Tabla 3: Resumen. Indicador estrategias del proyecto

\begin{tabular}{lccc}
\hline \multirow{2}{*}{\multicolumn{1}{c}{ Indicador }} & \multicolumn{3}{c}{$\begin{array}{c}\text { Metodologías desarrollo de Data } \\
\text { Warehouse }\end{array}$} \\
\cline { 2 - 4 } & Kimball & Hefesto & SAS \\
\hline Cuantificar el tiempo & 2 & 3 & 2 \\
Definir ventajas y desventajas & 1 & 1 & 1 \\
TOTAL & $\mathbf{3}$ & $\mathbf{4}$ & $\mathbf{3}$ \\
\hline
\end{tabular}

Fuente: elaboración propia

Gráfico 9. Resumen. Indicador estrategias del proyecto

Estrategias del proyecto

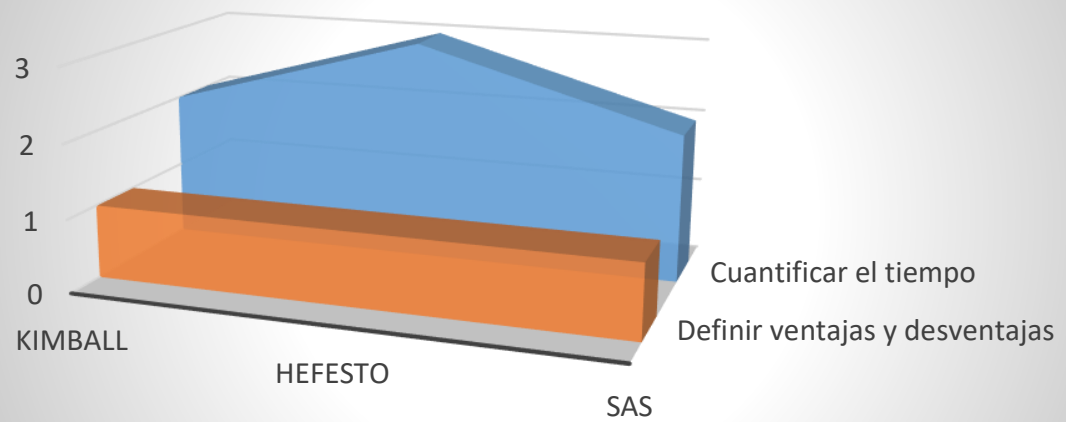

- Definir ventajas y desventajas

- Cuantificar el tiempo

Fuente: elaboración propia

\section{Fase 3: Planificación del proyecto}

Tabla 4: Resumen. Indicador planificación del proyecto

\begin{tabular}{lccc}
\hline \multirow{2}{*}{ Indicador } & \multicolumn{3}{c}{$\begin{array}{c}\text { Metodologías desarrollo de Data } \\
\text { Warehouse }\end{array}$} \\
\cline { 2 - 4 } & Kimball & Hefesto & SAS \\
\hline Cronograma de actividades & 3 & 1 & 3 \\
Análisis de riesgo & 1 & 3 & 3 \\
Definición de responsabilidades y grupo de & 3 & 3 & 1 \\
trabajo & $\mathbf{7}$ & $\mathbf{7}$ & $\mathbf{7}$ \\
TOTAL & & & \\
\hline
\end{tabular}

Fuente: Elaboración propia 
Gráfico 1. Resumen. Indicador planificación del proyecto

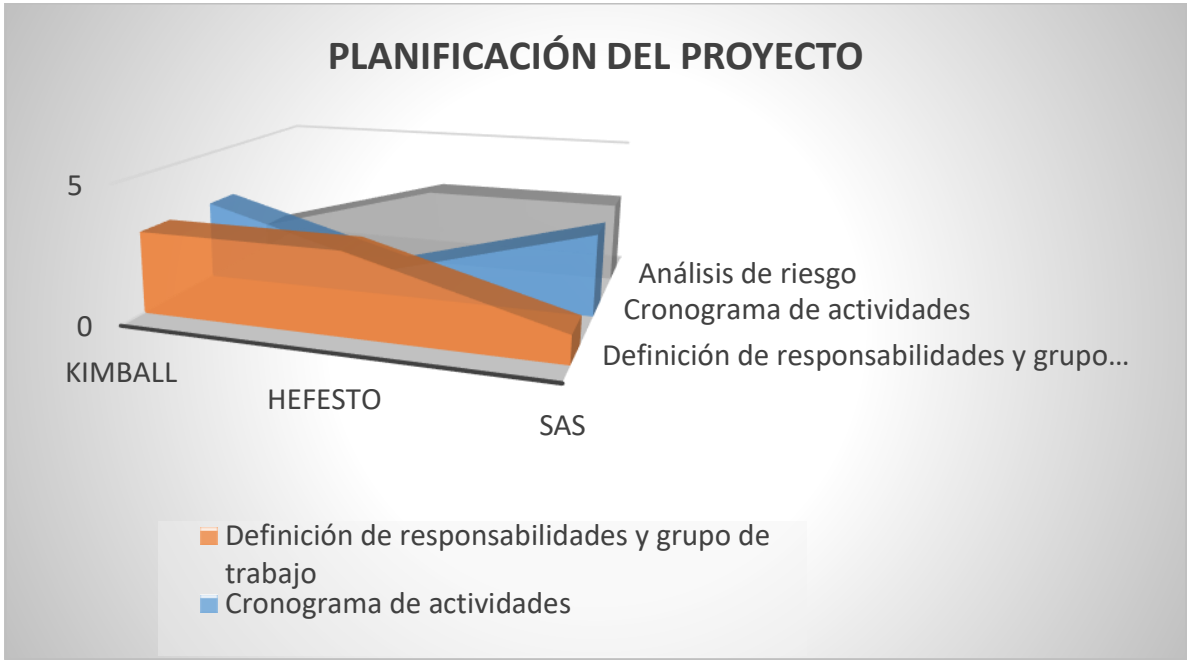

Fuente: elaboración propia

\section{Fase 4: Selección de la tecnología}

Tabla 5: Resumen. Indicador selección de la tecnología

\begin{tabular}{lccc}
\hline \multirow{2}{*}{ Indicador } & \multicolumn{3}{c}{$\begin{array}{c}\text { Metodologías desarrollo de Data } \\
\text { Warehouse }\end{array}$} \\
\cline { 2 - 4 } & Kimball & Hefesto & SAS \\
\hline $\begin{array}{l}\text { Entorno actual tecnológico de la } \\
\text { organización }\end{array}$ & 3 & 1 & 2 \\
Definición de tecnologías & 2 & 2 & 1 \\
TOTAL & $\mathbf{5}$ & $\mathbf{3}$ & $\mathbf{3}$ \\
\hline
\end{tabular}

Gráfico 2. Resumen. Indicador selección de la tecnología

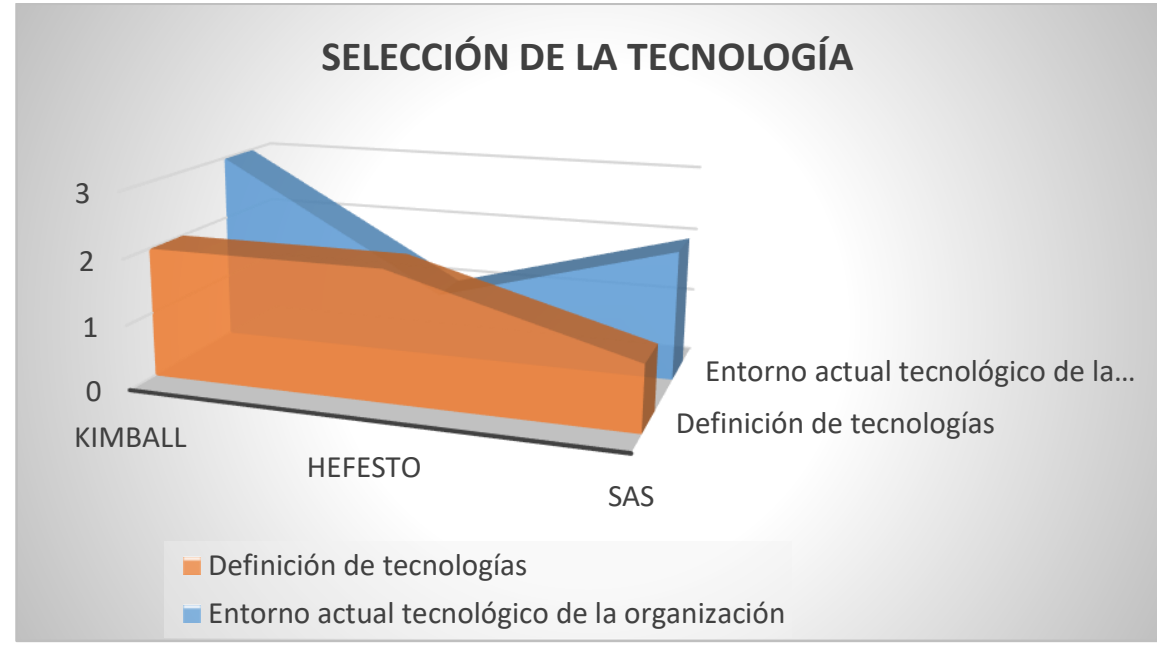

Fuente: elaboración propia 


\section{Fase 5: Diseño del sistema de información}

Tabla 6: Resumen. Indicador diseño del sistema

\begin{tabular}{lccc}
\hline \multicolumn{1}{c}{ Indicador } & \multicolumn{3}{c}{ Metodologías desarrollo de Data warehouse } \\
& Kimball & Hefesto & SAS \\
\hline Determinación del modelo de información & 2 & 3 & 1 \\
Diseño de la interfaz de usuario & 1 & 1 & 1 \\
TOTAL & $\mathbf{3}$ & $\mathbf{4}$ & $\mathbf{2}$ \\
\hline
\end{tabular}

Fuente: elaboración propia

Gráfico 3. Resumen. Indicador diseño del sistema

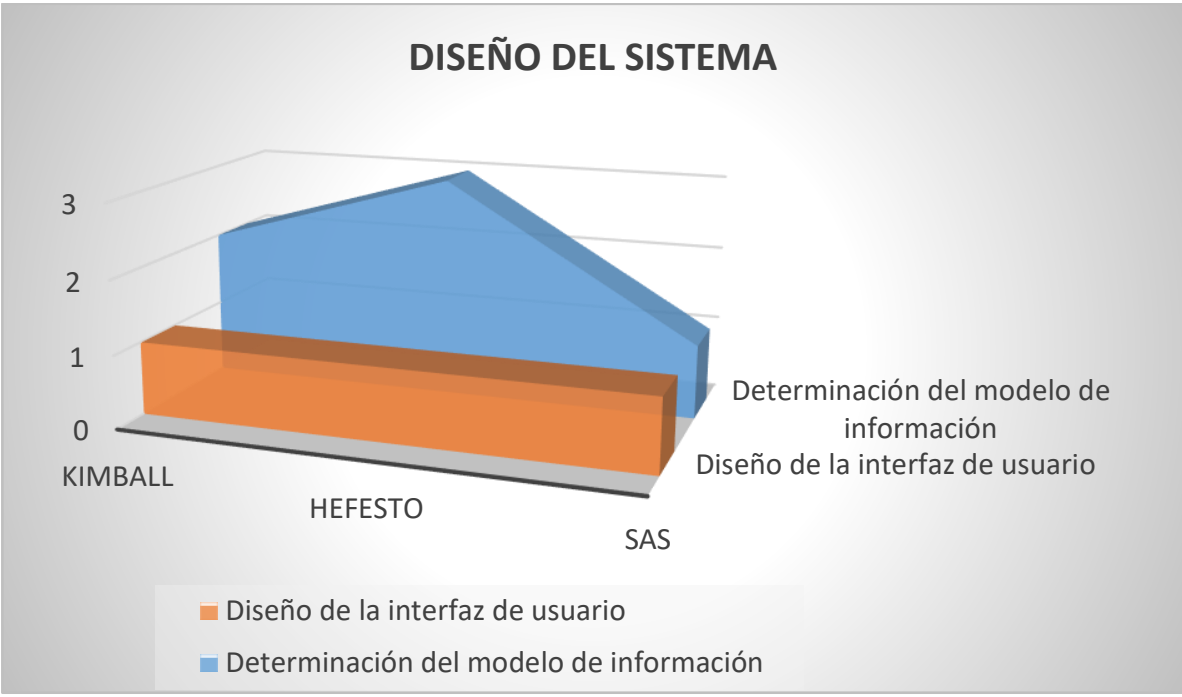

Fuente: elaboración propia

Fase 6: Elaboración del sistema de información

Tabla 7: Resumen. Indicador elaboración del sistema de información

\begin{tabular}{lccc}
\hline \multirow{2}{*}{\multicolumn{1}{c}{ Indicador }} & \multicolumn{3}{c}{$\begin{array}{c}\text { Metodologías desarrollo de Data } \\
\text { Warehouse }\end{array}$} \\
\cline { 2 - 4 } & Kimball & Hefesto & SAS \\
\hline Análisis de requerimientos (indicadores) & 3 & 3 & 1 \\
Modelo conceptual & 3 & 3 & 3 \\
Análisis OLTP & 3 & 2 & 1 \\
Conformar indicadores & 2 & 3 & 1 \\
Nivel de granularidad & 3 & 3 & 3 \\
Nivel conceptual ampliado & 1 & 3 & 1 \\
\hline
\end{tabular}


ISSN: 2602-8085

Modelo lógico de la estructura del DW

Tablas de dimensiones

Tabla de hechos

Proceso ETL

\begin{tabular}{ccc}
3 & 3 & 3 \\
3 & 3 & 3 \\
3 & 3 & 3 \\
3 & 3 & 3 \\
$\mathbf{2 7}$ & $\mathbf{2 9}$ & $\mathbf{2 2}$ \\
\hline
\end{tabular}

Fuente: Elaboración propia

Gráfico 4. Resumen. Indicador elaboración del sistema de información

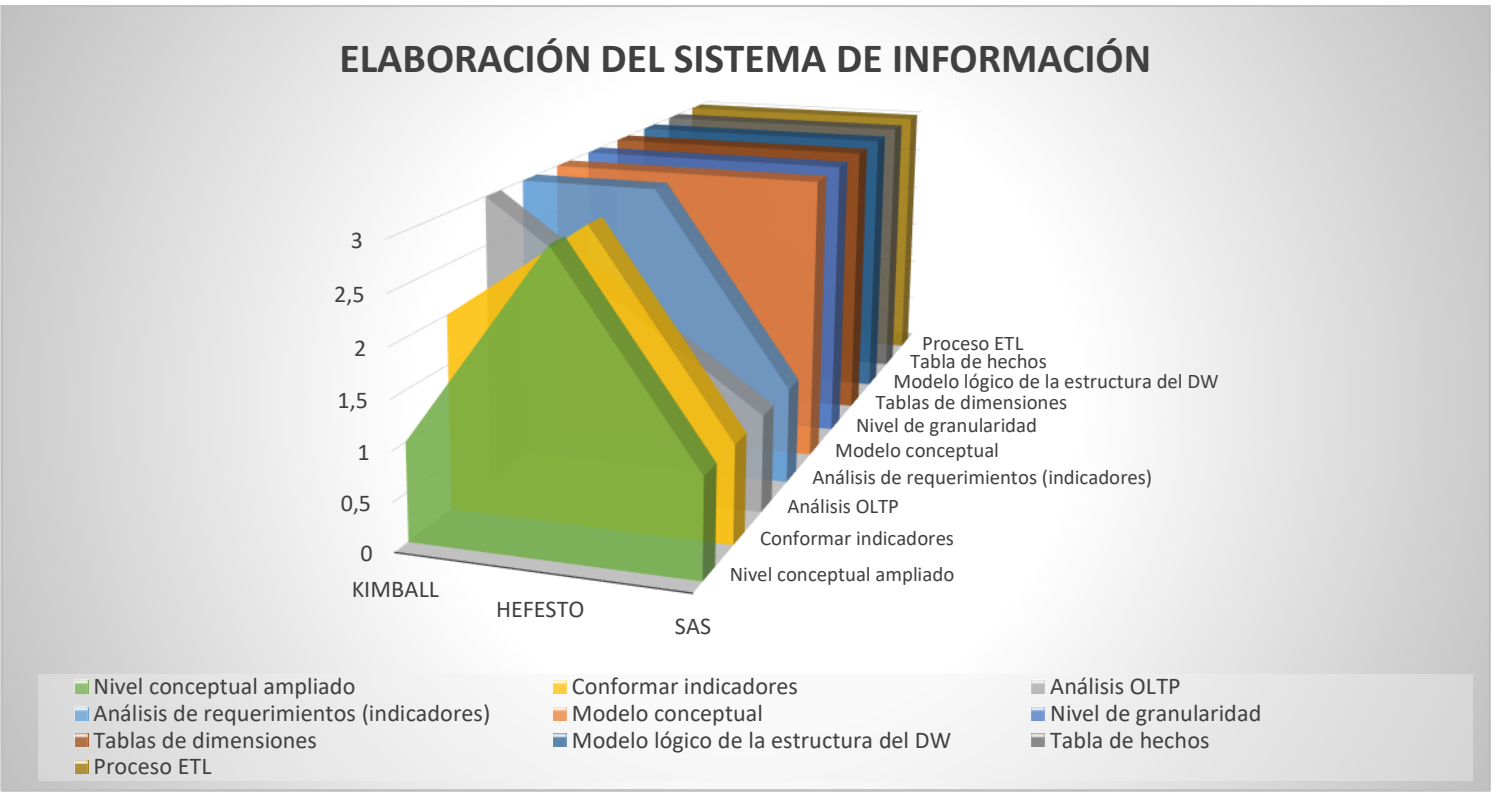

Fuente: elaboración propia

Determinación de parámetros de comparación de características metodológicas

\section{Características}

El análisis de las metodologías de desarrollo de un Data Warehouse debe ser complementada mediante un estudio de sus características generales, tomadas de Análisis, Diseño, Construcción e Implementación de un Data Warehouse para Toma de Decisiones y Construcción de los KPI, para la Empresa Kronosconsulting Cia Ltda.; de acuerdo al desarrollo del proyecto sobre el cual es implementado, estas mediciones facilitan una valoración estandarizada por lo que nos permite su análisis, de acuerdo a los parámetros de evaluación pre establecidos; para la valoración de las diferentes magnitudes tales como: el grado del cumplimiento de los objetivos, la capacidad de mejoras y del grado de satisfacción de los directivos. 
A continuación, se presenta el resumen de la valoración del indicador características metodológicas.

Tabla 108: Resumen. Indicador características metodológicas

\begin{tabular}{lccc}
\hline \multirow{2}{*}{\multicolumn{1}{c}{ Indicador }} & \multicolumn{3}{c}{$\begin{array}{c}\text { Metodologías desarrollo de Data } \\
\text { Warehouse }\end{array}$} \\
\cline { 2 - 4 } & Kimball & Hefesto & SAS \\
\hline Adaptable sobre cualquier metodología & 3 & 3 & 3 \\
Afinidad con el sistema actual & 3 & 3 & 3 \\
Comunicación con el cliente & 3 & 3 & 2 \\
Tamaño del proyecto & 2 & 3 & 2 \\
Tiempo en el análisis y diseño & 1 & 3 & 1 \\
Tiempo en construcción & 1 & 3 & 2 \\
Fácil entendimiento principiantes & 1 & 3 & 1 \\
Documentación Precisa & 3 & 3 & 2 \\
Más usada en el mundo & 1 & 3 & 1 \\
TOTAL & $\mathbf{1 8}$ & $\mathbf{2 7}$ & $\mathbf{1 7}$ \\
\hline
\end{tabular}

Fuente: elaboración propia

Gráfico 5. Parámetro. Características metodológicas

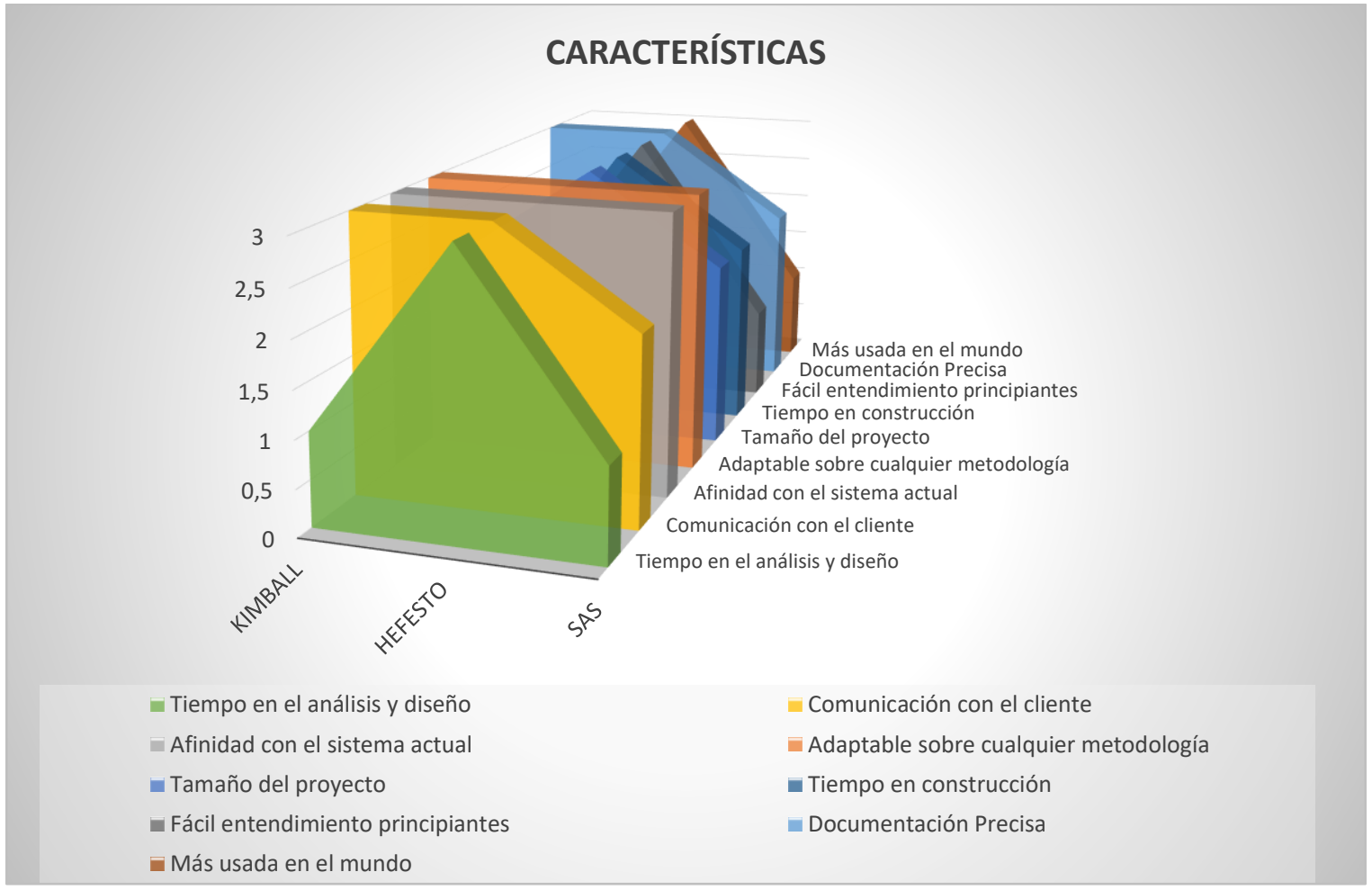

Fuente: elaboración propia 


\section{Análisis de resultados}

Resultados de comparativas fases metodológicas

Al analizar las metodologías Kimball, Hefestos y SAS con cada uno de los parámetros de comparación de las fases metodológicas se observa que la metodología Hefestos cumple con la mayoría de los índices tomados en cuenta de cada uno de los indicadores expuestos en las 6 fases examinadas obteniendo el primer lugar con un total de 54 puntos en la evaluación; mientras que la metodología Kimball cumple parcialmente con los indicadores obteniendo 52 puntos; por otro lado SAS obtiene una puntuación baja con 42 puntos, por lo que es la metodología con la menor posibilidad de uso para el desarrollo.

Resultados de comparativas características metodológicas

Al analizar las características con las métricas correspondientes a las metodologías Kimball, Hefestos y SAS se observa que la metodología Hefestos brinda una mayor cohesión en la construcción del Data Warehouse cumpliendo con el mayor porcentaje de los indicadores establecidos para dicha evaluación haciéndola la metodología con mayor valoración al obtener 29 puntos; en segundo lugar, tenemos la metodología Kimball con 27 puntos la cual no cumple con algunas de las características valoradas; mientras que la metodología SAS se encuentra en el último lugar.

\section{Conclusiones}

- La investigación de los principios teóricos de las metodologías Kimball, Hefestos y SAS, se han convertido en el sustento para el desarrollo de soluciones Data Warehouse, gracias a la rapidez y sencillez en la obtención de reportes.

- La selección de los parámetros de comparación de las metodologías a emplearse en un Data Warehouse, se desarrollaron en base a la investigación del número de fases requeridas para la elaboración de un Data Warehouse, así como en las características que brindan las metodologías Kimball, Hefestos y SAS a un proyecto pequeño, permitió conocer que la metodología Hefestos, proporciona una identificación ágil de los objetivos y resultados del proyecto para garantizar la toma de decisiones.

\section{Referencias bibliográficas.}

BERNABEU, D. (2009). Businness Intelligence. Obtenido de http://www.dataprix.com/datawarehousing-y-metodologia-hefesto/1-business-intelligence/14-beneficios

BERNABEU, R. (2010). Obtenido de Data warehousing y metodología Hefesto: http://www.dataprix.com/es/data-warehousing-hefesto 
COMPUTACIÓN. (11 de 2010). Datos e Información. Obtenido de http://compuah.blogspot.com/2010/11/datos-e-informacion.html

CUELLAR, G. (2011). Data warehouse, aspectos técnicos, caracteristicas, usos, beneficos, componentes, herramientas OLAP. Obtenido de http://fccea.unicauca.edu.co/old/datawarehouse.htm

DEVLIN, B. (2011). The data-driven Business and other Lessons from History. Obtenido de http://assets.en.oreilly.com/1/event/55/The\%20Datadriven $\% 20$ Business $\% 20$ and $\% 20$ Other\%20Lessons\%20from\%20History\%20Presentation.p df

ESPINOSA, R. (04 de 2010). Descubriendo el business intelligence. Obtenido de Rincon del BI: https://churriwifi.wordpress.com/2010/04/

FERNÁNDEZ, C. (2015). Fases de implantación de un data warehouse. Obtenido de $\mathrm{http} / / / \mathrm{www}$.dataprix.com/fases-de-implantacion-de-un-data-warehouse

GARCÉS, M. (2015). Metodologías e implmentación de alternativas busisness intelligence opensource vs. propietarias en entornos tradicionales; caso prototipo en las pymes en el sector agroindustrial (Tesis de postgrado). Universidad de las Américas. Obtenido de http://dspace.udla.edu.ec/bitstream/33000/2660/1/UDLA-EC-TMGSTI-2015-01.pdf

KIMBALL, G. (2016). Kimball group. Obtenido de http://www.kimballgroup.com/data-warehousebusiness-intelligence-resources/books/kimball-reader/

KIMBALLL/INMON. (2012). Enfoques de desarrollo DW. Obtenido de http://www.interaktiv.cl/blog/wp-content/uploads/2012/04/4.Metodologia_disegno_DW1.pdf

LEÓN, M. (2014). Ciclo de vida de Ralph Kimball. Obtenido de http://luisleonin.blogspot.com/2014/02/ciclo-de-vida-de-ralph-kimball.html

LEÓN, M. (02 de 2017). Inteligencia de Negocio. Obtenido de Ciclo de vida de Raplh Kimball: http://luisleonin.blogspot.com/2014/02/ciclo-de-vida-de-ralph-kimball.html

LOZADA X \& CRUZ, H. (2014). Análisis, diseno, construcción e implementación de una data warehouse para la toma de decisiones y construcción de los KPI, para la empresa KRONOSCONSULTING CIA LTDA. (Tesis de pregrado). Escuela Superior Polítecnica del Ejército. Obtenido de http://repositorio.espe.edu.ec/xmlui/bitstream/handle/21000/8027/TESPE-047750.pdf?sequence $=1 \&$ isAllowed $=\mathrm{y}$

MURILLO, C. (2008). Implemetación de un Data Mart para el ramo de vehículos de una empresa aseguradora (Tesis de pregrado). Escuela Politécnica Nacional. Obtenido de http://bibdigital.epn.edu.ec/handle/15000/1281

PEÑA J \& SÚAREZ, J. (2008). Utilización de infoemación histórica para desiciones empresariales. (Tesis de pregrado). Pontificia Universidad Javeriana. Obtenido de http://hermes.javeriana.edu.co/biblos/tesis/ingenieria/Tesis204.pdf

REDIFP. (2013). Catálogo de indicadores para la toma de decisiones de los Institutos de Formación Profecional.

Obtenido de http://www.ilo.org/wcmsp5/groups/public/---americas/---ro-lima/---sro -san_jose/documents/publication/wcms_243198.pdf

ROJAS, A. (2014). Implementación de un Data Mart como solución de inteligencia de negocios, bajo la metodología de Ralph Kimball para optimizar la toma de desiciones en el departamento de finanzas de la contraloría (Tesis de pregrado). Universidad de San Martín de Porres. 
Obtenido

http://www.repositorioacademico.usmp.edu.pe/bitstream/usmp/1061/1/rojas_a.pdf

SINNEXUS. (2016). Business Intelligence Informática estratrégica. Obtenido de http://www.sinnexus.com/business_intelligence/

SINNEXUS. (2016). Datos, formación, conocimiento. Obtenido de http://www.sinnexus.com/business_intelligence/piramide_negocio.aspx

TURNERO, I. (2015). Documentación del SGC: el enfoque basado en procesos. Obtenido de http://www.monografias.com/trabajos97/documentacion-del-sgc-enfoque-basadoprocesos/documentacion-del-sgc-enfoque-basado-procesos.shtml

UVIDIA, M. (2016). Descubrimiento de conocimiento en base de datos para la toma de decisiones en la unidad de nivelación y admición de la ESPOCH. (Tesis de postgrado). Escuela Superior Politécnica de Chimborazo.

Obtenido

de http://repositorio.pucesa.edu.ec/bitstream/123456789/1601/1/76134.pdf

VICENTE, A. (03 de 2017). Dato, Información y Conocimiento. Obtenido de http://tecnowalsh.blogspot.com/

VILLAREAL, R. (2013). Estudio de metodologías de Data Warehouse para la implementación de repositorios de información para la toma de decisiones gerenciales (Tesis pregrado). Universidad Técnica Norte. Obtenido de https://www.google.com/url?sa=t\&rct=j\&q=\&esrc=s\&source=web\&cd=6\&cad=rja\&uact= 8\&ved=0ahUKEwjr0cGz1IPXAhWKMyYKHSIbDvQQFgg5MAU\&url=http\%3A\%2F\%2 Frepositorio.utn.edu.ec\%2Fbitstream\%2F123456789\%2F7015\%2F1\%2F04\%2520RED\%2 520080\%2520TRABAJO\%2520GRADO.pdf\&usg=AOvVa 


\section{PARA CITAR EL ARTÍCULO INDEXADO.}

Silva Peñafiel, G., Zapata Yánez, V., Morales Guamán, K., \& Toaquiza Padilla, L. (2019). Análisis de metodologías para desarrollar Data Warehouse aplicado a la toma de decisiones. Ciencia Digital, 3(3.4.), 397-418. https://doi.org/10.33262/cienciadigital.v3i3.4.922

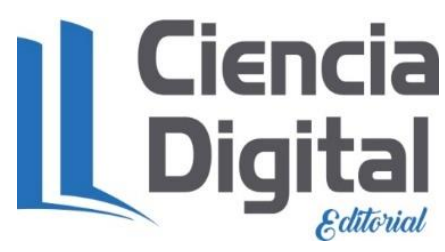

El artículo que se publica es de exclusiva responsabilidad de los autores y no necesariamente reflejan el pensamiento de la Revista Ciencia Digital.

El artículo queda en propiedad de la revista y, por tanto, su publicación parcial y/o total en otro medio tiene que ser autorizado por el director de la Revista Ciencia Digital.
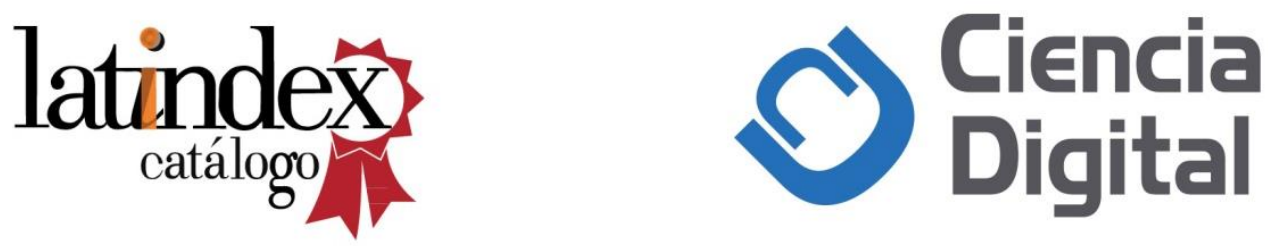